\title{
Evaluación del impacto de la capacitación a directivos de la provincia de
}

\section{Guantánamo}

\author{
Evaluation of the impact of management training in the province of Guantánamo \\ Avaliação do impacto da formação de gestores na provincia do Guantánamo
}

\section{Resumen}

Las organizaciones empresariales se enfrentan en estos tiempos a cambios acelerados que se producen en todos los ámbitos de la sociedad; razón por lo cual la capacitación de los recursos humanos - y especialmente de los que dirigen - con enfoque sistémico, es una condición imprescindible para elevar su calificación directiva especializada. Esto presupone que la evaluación de la capacitación constituya un recurso para validar su perfeccionamiento constante y de esa forma distinguir puntualmente la situación que presenta verificando el cumplimiento de los objetivos, los resultados, efectos e impactos. Por estas razones la investigación realizada tuvo como objetivo la elaboración de un sistema de indicadores para evaluar el impacto de la capacitación a directivos en la Provincia Guantánamo, teniendo como base el Diplomado de Gerencia Empresarial y Administración Pública, el cual resultó evaluado de alto impacto una vez aplicado lo planteado. Esta investigación constituye un material de consulta para profesores, metodólogos y directivos porque en ella se ofrecen instrumentos para evaluar el impacto de la capacitación de los directivos a partir de un sistema de indicadores. Los métodos de investigación empleados fueron: analítico-sintético, inductivodeductivo, histórico-lógico, estadístico-matemático, las encuestas, el estudio de documentos, y el criterio de expertos, los cuales posibilitaron evaluar las tendencias y enfoques existentes referidos al tema objeto de estudio; así como enriquecer, completar y contrastar información sobre el impacto de la preparación recibida. Igualmente permitió valorar la coherencia y factibilidad de la propuesta de indicadores para su utilización con el fin de perfeccionar la capacitación como proceso.

Palabras clave: Evaluación; Impacto; Capacitación; Directivos.

\begin{abstract}
Business organizations are currently facing accelerated changes that occur in all areas of society; for this reason, the training of human resources - and especially those who direct - with a systemic approach, is an essential condition to upgrade their specialized managerial qualification. This presupposes that the valuation of training constitutes a resource to validate its constant improvement, and in this way to punctually distinguish the situation it presents, verifying the fulfillment of the objectives, results, effects and impacts. For these reasons, the objective of the research carried out was to develop a system of indicators to evaluate the impact of training for executives in Guantanamo's Province, based on a Diploma in Business Management and Public Administration, which was evaluated with high impact once applied what was previously stated. This research constitutes a reference material for teachers, methodologists and managers because it offers instruments to evaluate the impact of training managers based on a system of indicators. The research methods used were: analytical-synthetic, inductive-deductive, historical-logical, statistical-mathematical, surveys, the study of documents, and the criteria of experts, which made it possible to evaluate the existing trends and approaches related to the subject matter study; as well as enriching, completing and contrasting information on the impact of the preparation received. It also made possible to assess the coherence and feasibility of the proposed indicators used in order to improve training as a process.
\end{abstract}

Keywords: Evaluation, Impact, Training, Executives.

\section{Resumo}

As organizações empresariais estão enfrentando mudanças aceleradas que ocorrem em todas as áreas da sociedade; Por isso, a formação de recursos humanos e principalmente daqueles que administram com enfoque sistémico, é condição essencial para elevar sua qualificação gerencial especializada. Isto pressupõe que a avaliação da formação 
constitui um recurso para validar a sua melhoria constante e desta forma distinguir pontualmente a situação que apresenta, verificando o cumprimento dos objetivos, resultados, efeitos e impactos. Por estes motivos, o objetivo da pesquisa realizada foi desenvolver um sistema de indicadores para avaliar o impacto da formação de gestores na Província de Guantánamo, com base no Diploma em Gestão de Negócios e Administração Pública, que foi avaliado como de alto impacto uma vez aplicado o que foi tratado. Esta pesquisa constitui um material de referência para professores, metodólogos e gestores, pois oferece instrumentos para avaliar o impacto da formação gerencial a partir de um sistema de indicadores. Os métodos de investigação utilizados foram: analítico-sintético, indutivo-dedutivo, histórico-lógico, estatístico-matemático, inquéritos, estudo de documentos e critérios de especialistas, que permitiram avaliar as tendências e abordagens existentes relacionadas com o assunto. Estudo; bem como enriquecer, completar e contrastar informações sobre o impacto da preparação recebida. Também possibilitou avaliar a coerência e a exequibilidade dos indicadores propostos para uso, a fim de melhorar o treinamento como processo.

Palavras-chave: Avaliação; Impacto; Treinamento; Gestores.

\section{Introducción}

El mundo actual está lleno de complejidades que evidencian cada vez más que la capacitación de los recursos humanos con enfoque sistémico es condición ineludible para que la humanidad pueda enfrentar los retos y desafíos que imponen los cambios acelerados que se dan en todos los ámbitos de la sociedad. Este reto al desarrollo de la política estatal resulta una prioridad para aquellos que ejercen alguna de estas tareas directivas, para la cual existen en Cuba determinadas estrategias sectoriales, cuya razón de ser es garantizar la formación continua de todos los directivos para dotarlos de conocimientos, habilidades y capacidades que les permitan conducir colectivos cohesionados, eficientes, competentes y comprometidos con los resultados de su organización.

Si lo anterior es válido para toda la humanidad, en el transcurso de estos últimos años Cuba se ha visto envuelta en cambios trascendentes que conllevan a transformaciones socioeconómicas: se han introducido conocimientos científicotecnológicos y se han desarrollado habilidades gerenciales no potenciadas nunca antes y que -por lo tanto- precisan de perfeccionamiento y actualización sistemática.

Este perfeccionamiento presupone tener en cuenta los principios que rigen la gerencia moderna. Entre ellos cabe destacar el enfoque estratégico de la dirección, el trabajo con los recursos humanos (como elemento estratégico más importante) y el fin primario de las organizaciones: disponer de una visión futurista conociendo las motivaciones personales y grupales; estas son las guías fundamentales para desarrollar el trabajo de gestión empresarial.

Los procesos de participación social interactiva con los trabajadores implican el éxito en la toma de decisiones y en la solución de los problemas, que así les son cada vez más inmediatos, cotidianos y determinantes. En consecuencia, el clima laboral es cada vez más flexible, integral, innovativo y creativo: porque el control se calibra más en los resultados que en los procesos. De ahí que la concepción actual no sea dirigir a los hombres, sino dirigir con los hombres.

El punto de partida para acometer todo el proceso de perfeccionamiento organizacional deberá guiar la misión y los fines de cada organización. Estos fines actúan como legitimadores de la acción, como patrones de la evaluación (efectividad y eficiencia) y como regla de estudio. Su carácter legitimador, garantiza la integración del orden institucional.

Por otra parte, el sistema de valores y creencias fundamentales que determinan la misión organizacional establece las metas a corto y largo plazo, y los objetivos mediatos e inmediatos que influyen sobre una gama de actitudes expresadas en los diferentes niveles estructurales de la compañía, marcando el rumbo de todas las actividades e imprimiéndole una orientación permanente en el sentido previsto.

El estado cubano cuenta con los mejores activos económicos, provenientes de su capital humano: el talento, la inteligencia, la calidad humana y las cualidades de sus hombres y mujeres; por consiguiente, la formación y desarrollo de los que tienen la función de conducir los recursos humanos hacia el logro de superiores objetivos socioeconómicos sigue siendo una tarea prioritaria, máxime si tiene en cuenta que los directivos -a todos los niveles- deben estudiar tenaz y sistemáticamente, 
para que aspiren a dominar las más modernas y eficientes tecnologías, a mantenerse informados de la situación económica, política y sociocultural (de Cuba y del mundo) y a combatir permanente por alcanzar los más altos niveles de excelencia.

Por todo lo anteriormente referido, en la Estrategia Nacional de Preparación y Superación de los directivos del Estado y del Gobierno, se concibe la preparación de estos a partir del concepto de su integridad y organización sistémica, contemplando todos los componentes y combinando todas las formas, los contenidos, los plazos temporales, y los recursos necesarios para que sea continua, eficiente y específica; dirigida a las necesidades personales de cada directivo y al cumplimiento de los planes de desarrollo de cada organismo, territorio o entidad.

En los informes nacionales de Balance de la Preparación y Superación de los directivos de los últimos años e expresa la aceptación de esta Estrategia, pero todavía existen muy pocas acciones de retroalimentación y algunas experiencias muy aisladas, y no se poseen aún métodos, ni herramientas adecuadas para medir el impacto de esta actividad de capacitación.

Por tal motivo el Ministerio de Educación Superior ha convocado al personal docente de todas las universidades cubanas a investigar en torno a la elaboración de una metodología que -validada adecuadamente- permita medir el impacto de la capacitación que reciben los directivos del país.

En consonancia con ello en la provincia de Matanzas (Universidad Camilo Cienfuegos) se han desarrollado dos Talleres Nacionales de Medición del Impacto de la Capacitación (febrero del 2003 y febrero del 2004), eventos donde se han presentado varias ponencias que abordaron esta temática. En ninguno de los trabajos presentados se aportaron indicadores que permitan evaluar el impacto de la capacitación a directivos.

En las búsquedas efectuadas a través de la Red Mundial de Información se hallaron diversas recomendaciones metodológicas que no se relacionan en lo absoluto con el tema abordado en esta investigación, por lo que a tenor de estos resultados y verificada la existencia de esta dificultad práctico-metodológica para evaluar el impacto de la capacitación en los directivos y teniendo en cuenta los empeños y otras aproximaciones logradas, cabe plantearse el siguiente problema científico:

No existe un sistema de indicadores que permita evaluar el impacto de la capacitación a directivos en la provincia Guantánamo.

Se considera que por la intencionalidad que se persiguió con la indagación que la misma adquiere el carácter de investigación cualitativa. Eso corrobora el factor de pretender en primera instancia diagnosticar el estado real de la preparación que alcanzan los directivos capacitados en la provincia de Guantánamo en el ulterior desempeño de sus funciones para evaluar el impacto de dicha capacitación a partir de la elaboración de un sistema indicadores.

Según el objetivo es descriptivo; el apoyo de las diferentes bibliografías sobre el tema investigado, dentro de los que se puede mencionar textos de diferentes autores, revistas, trabajos científicos y publicaciones .La consulta y revisión de todo este material sirvió de base para el marco teórico de este trabajo.

La capacitación a directivos es un problema actual y un reclamo de la dirección del estado cubano a las universidades y escuelas ramales en cuanto a la capacitación sistemática, a partir de las necesidades y condiciones, del entorno socioeconómico y político, en que se desenvuelven estas entidades.

El diseño y elaboración del sistema de capacitación a directivos es una actividad especializada dirigida a profundizar en los conocimientos, y el desarrollo de capacidades y habilidades para que éstos cumplan con efectividad sus funciones.

Un sistema es un todo con cualidades propias que no la poseen ninguna de las partes o elementos que lo conforman, existe una estrecha relación entre esas partes y el medio. En un sistema integral de capacitación se puede encontrar ese todo con cualidades intrínsecas que revelan el nexo entre sus componentes, relaciones específicas entre necesidades de capacitación y los objetivos; entre los objetivos y los programas, los métodos seleccionados, las formas organizativas de cada actividad, los contenidos y la forma de evaluación. Se distingue el hombre, que en este caso es a la vez sujeto y objeto de la capacitación. 


\subsection{Peculiaridades de la capacitación a directivos.}

Todo proceso de enseñanza aprendizaje es complejo y tiene como propósito la incorporación de nuevos conocimientos, hábitos y habilidades de los capacitados, por lo que se manifiestan contradicciones lógicas entre lo que se conoce y lo que se está por conocer.

\section{Metodología para la Obtención de los Indicadores de Evaluación de Impacto}

En la determinación del sistema de indicadores para evaluar el impacto de la capacitación se parte de la concepción pedagógica de la capacitación, teniendo en cuenta los conceptos de evaluación, impacto e impacto de la capacitación.

Para la realización de la evaluación se han diferenciado una serie de fases que se exponen a continuación y que son la base del sistema de indicadores propuesto. Estas fases -aunque tienen cierto orden lógico- no tienen por qué ejecutarse por este cronograma ya que en algunos casos las fases son complementarias y hay que realizarlas simultáneamente y otros, son excluyentes o consecutivas en su realización temporal.

En las fases de evaluación del proceso de capacitación se tuvieron en cuenta los siguientes aspectos o vías para su análisis: diagnóstico de las necesidades de aprendizaje, verificación in situ de la preparación inicial de los cursistas, evaluación de conocimientos y habilidades adquiridas durante el proceso de capacitación - control concurrente, operativo o a de interin -, evaluación de conocimientos y habilidades adquiridas en toda la capacitación, tanto al finalizarla como pasado cierto tiempo convencional o apropiado - control al final y control después del final del curso-.

Se efectuó un estudio descriptivo y analítico para evaluar le calidad de las fases de planificación, ejecución, resultado e impacto de los programas de capacitación que se imparten que constituyen la base del sistema de indicadores propuesto.

En la fase de evaluar la planificación de la capacitación se consideran todas las acciones que se realizan con anterioridad al montaje de los programas con el objetivo de evaluar el diseño y montaje de dichos programas y determinar el nivel de conocimiento de los destinatarios sobre los temas que se abordan en los mismos al ser impartidos: Entre estas acciones se tiene: definición de la población a estudiar, análisis del diagnóstico de las necesidades del aprendizaje, recepción de la información primaria y secundaria, elaboración de los cuestionarios, entrevistas y encuestas, así como la especificación de los programas de capacitación a evaluar.

Con la fase evaluativa de ejecución de la capacitación recibida se estableció la disponibilidad y calidad de la infraestructura material y de los medios didácticos de los programas, así como se pudo comprobar la calidad de las actividades docentes que se imparten y el criterio de los cursistas sobre la calidad de la ejecución de los programas de capacitación que reciben.

En la fase evaluativa de los resultados de la capacitación que reciben, se analizan dos momentos fundamentales: al finalizar -efecto final- y después de culminar dicho proceso, o sea, el impacto.

Con el fin de conformar la base de la propuesta de los indicadores se estableció previamente en un cuadro donde se recogen: Variables, indicadores.

Se hizo un análisis de las diferentes variables existentes que permitan medir impacto y resultaron seleccionadas las siguientes: Contenido, claustro de profesores, cursistas y métodos y medios empleados.

En función de los criterios de evaluación adoptados y las características de las áreas escogidas, como objeto de aplicación para apreciar la efectividad de la capacitación de los cursos recibidos y a partir de un amplio menú de posibles indicadores -tomados de fuentes especializadas o especialmente elaboradas surgió la propuesta del sistema de indicadores, conformado por indicadores (Quadro 1). 
Quadro 1 - Proposto del sistema de indicadores.

\begin{tabular}{|c|c|}
\hline Variables & Sistema de Indicadores \\
\hline \multirow{3}{*}{ 1. Contenido } & 1.1 Coherencia en la estructuración de los módulos \\
\hline & $\begin{array}{l}\text { 1.2 Correspondencia del diseño del Diplomado con las principales necesidades de los } \\
\text { cursistas. }\end{array}$ \\
\hline & 1.3 Nivel de satisfacción de los cursistas con la calidad de la preparación recibida. \\
\hline \multirow{5}{*}{$\begin{array}{c}\text { 2. Claustro } \\
\text { de } \\
\text { profesores }\end{array}$} & 2.1 Calidad del proceso \\
\hline & 2.2 Estabilidad del claustro. \\
\hline & 2.3 Categoría docente principal \\
\hline & 2.3.1 Titular y Auxiliar \\
\hline & 2.4 Título académico y científico \\
\hline \multirow{5}{*}{ 3. Cursistas } & 3.1 Calidad del ingreso. \\
\hline & 3.2 Aprovechamiento Académico \\
\hline & 3.3 Promovidos a cargos de dirección \\
\hline & 3.4 Desempeño profesional \\
\hline & $\begin{array}{l}3.5 \text { Nivel de satisfacción de los empleadores con la calidad de la preparación mostrada } \\
\text { por los egresados en el desempeño de sus funciones. }\end{array}$ \\
\hline \multirow{3}{*}{$\begin{array}{l}\text { 4. Métodos } \\
\text { y medios } \\
\text { Empleados }\end{array}$} & 4.1 Empleo la Dirección participativa \\
\hline & 4.2 Utilización de situaciones Problemáticas \\
\hline & 4.3 Utilización de las Tecnologías de la información y las comunicaciones ( TIC ) \\
\hline
\end{tabular}

Fuente: Autores.

\section{1 Contenido}

\subsubsection{Coherencia en la estructuración de los módulos}

Es la coherente articulación de los módulos y los cursos de postgrado que forman parte de cada módulo. Que éstos tenga correctamente definido las temáticas, horas clases, actividades y el cumplimiento de los objetivos específicos, los cuales deben responder a los generales del curso como consecuencia de su derivación gradual.

\subsubsection{Correspondencia del diseño del Diplomado con las principales necesidades de los cursistas.}

Para garantizar el adecuado diseño del proceso de enseñanza aprendizaje es necesario diagnosticar las necesidades de aprendizaje de los cursistas, pues asegura una adecuada formulación de los objetivos e información a los profesores encargados de la capacitación, para que al término de este proceso se satisfagan las exigencias que motivaron la realización del programa, partiendo de las exigencias laborales y personales que se le plantean respectivamente.

\subsubsection{Nivel de satisfacción de los egresados con la calidad de preparación recibida.}

Los cursistas manifiestan altos niveles de satisfacción con la preparación alcanzada en el curso en elementos relacionados con los componentes principales definidos para la capacitación de los directivos.

\subsection{Claustro de professores}

\subsubsection{Satisfacción con el proceso.}

La universidad debe proponerse saber cómo son acogidos, sentidos, comprendidos y apoyados los programas o planes de capacitación, por los profesionales y las organizaciones , y por otra parte, comprobar cómo esta responde a las expectativas y exigencias personales y organizacionales con acciones comprometidas, relevantes y oportunas. 


\subsubsection{Categoría académica y científica.}

En la comunidad universitaria es conveniente hablar de excelencia académica porque resultaría imposible garantizar la calidad de este nivel educacional si simultáneamente no se promoviera la excelencia investigativa, docente y laboral de los cursistas; es evidente que el anhelo de la excelencia reafirma la pertinencia de la integralidad, como factor esencial de la calidad.

\subsubsection{Estabilidad del claustro.}

El claustro de profesores previstos desde su concepción sean los que se mantengan en las diferentes ediciones porque esto garantiza la consolidación y profundización en los contenidos que se imparten, un mejor perfeccionamiento de los programas a partir de las experiencias que adquiere el profesor y aporta el cursistas como agente activo del proceso.

Constituye un requisito indispensable para que se alcance un determinado grado de desarrollo científico-tecnológico que este entre a formar parte indisoluble de la estrategia de los programas que se imparten, pues la interacción entre las exigencias, la forma de utilización de los recursos y la manera de organizar los diferentes componentes, definirán la estrategia pedagógica a seguir.

\subsection{Cursistas}

\subsubsection{Calidad del ingreso}

Las propuestas deben realizarse sobre la base de los resultados concretos alcanzados en el puesto de trabajo y la eficiencia en el desempeño de sus funciones de dirección. Además de cumplir con los requisitos para la selección.

\subsubsection{Aprovechamiento Académico}

El profesor tiene la obligación de concebir la evaluación del aprendizaje holísticamente, con sistematicidad e integralidad, para hacer de ella un instrumento que le permita analizar cómo los alumnos asimilan y sistematizan conceptos científicos, cómo aplican leyes y teorías en la manifestación de vínculos entre los diferentes hechos, procesos y fenómenos que ocurren en la realidad objetiva,. En fin una evaluación que le abra espacios de reflexión y mejoramiento a su proceso de enseñanza.

\subsubsection{Promovidos a cargos de dirección.}

Fueron promovidos a cargos de dirección por estar dotados de conocimientos, habilidades, capacidades y cualidades morales que les permitan dirigir colectivos cohesionados, eficientes, competentes, con firmes convicciones y comprometidos con los resultados de la organización que representan.

\subsubsection{Desempeño Profesional}

Alcanza resultados satisfactorios en el desempeño de sus funciones que se ven reflejados en el resultado de la organización donde labora.

\subsubsection{Nivel de satisfacción de los empleadores con la calidad de la preparación mostrada por los egresados en el} desempeño de sus funciones.

Los empleadores de las diferentes organizaciones a las cuales pertenecen los egresados expresan altos niveles de satisfacción con la preparación general integral ofrecida en la capacitación. 


\subsection{Métodos y medios Empleados}

\subsubsection{Empleo la Dirección participativa}

La capacitación grupal prevé la utilización de métodos participativos, demostrativos e individualizados y exige una cuota de responsabilidad de los participantes.

Aplica la concepción de dirigir con los hombres, por lo que los procesos de participación social interactiva con los trabajadores implican el éxito en la toma de decisiones y en la solución de los problemas que así les son cada vez más inmediatos, cotidianos y determinantes. En consecuencia, el clima laboral es cada vez más flexible, integral, innovador y de gran creatividad.

\subsubsection{Utilización de situaciones problemáticas.}

Favorece la formación de profesionales competentes para detectar problemas en la dirección de las organizaciones y darles la solución científica requerida a éstos. Además permite comprobar el grado de asimilación consciente de los conocimientos y habilidades para aplicarlos a situaciones semejantes o nuevas, para lo cual es indispensable que los cursistas dominen conceptos básicos, leyes o teorías; así como generalizaciones de las materias de enseñanza y de las relaciones que se establecen entre éstas.

\subsubsection{Utilización de las Tecnologías de la información y las comunicaciones (TIC)}

El empleo de las técnicas modernas de la dirección empresarial adecuándolas a las características de cada organización y basadas en las mejores y más avanzadas prácticas contemporâneas.

\subsection{Escala para evaluar cada indicador}

Antes de comenzar se considera conveniente a modo de aclaración plantear que se aplicó para la evaluación de los indicadores la siguiente escala:

1: Muy Bajo impacto, (sí alcanza menos de 3 como promedio);

2: Bajo impacto, (sí alcanza un 3 a 3,4 como promedio);

3: Impacto medio (sí alcanza un 3,5 a 3,9 como promedio);

4: Alto impacto, (sí alcanza un 4,0 a 4,4 como promedio);

5: Muy alto impacto, (sí alcanza un 4,5 a 5 como promedio).

En el sistema de indicadores propuesto para medir el impacto de la capacitación empleo una escala para evaluar por separado cada uno de los indicadores que componen la variable. El promedio de los indicadores se toma como valor que expresa el impacto que alcanza la variable analizada. Posteriormente se obtienen la media aritmética de los valores obtenidos en cada variable; El promedio así calculado es un índice general integral que ofrece una valoración del impacto de la capacitación en un solo valor, lo cual proporciona ventajas al disponer en una primera instancia del comportamiento, sin necesidad de analizar cada variable de forma independiente. Lo que no quiere decir que esto no pueda utilizarse para evaluar cada variable por separado con el propósito de detectar cuál de ellas ocasionó irregularidades en el proceso.

\section{Desarrollo}

La capacitación a directivos es un proceso que adquiere relevancia, pues estos poseen vivencias, saberes acumulados, experiencias de trabajo y de la propia vida, que los conducen a solucionar sistemáticamente diferentes problemáticas que 
tienen que ver con la gestión empresarial o cultura organizacional, lo que fortalece su conducta, y reafirma su personalidad y sus criterios. En este sentido, el significado que la práctica tiene como fuente del conocimiento, no puede ser obviado.

La capacitación es una forma de enseñanza que -apoyada en la práctica- persigue el adiestramiento, la recalificación y el perfeccionamiento de los directivos. Su ejecución se concibe en dos etapas: una primera consistente en la preparación o apropiación de los conocimientos básicos y las experiencias elementales necesarias para dirigir, y la segunda es la superación, que se concreta mediante la actualización, la complementación y ampliación de los conocimientos y habilidades de dirección.

\section{¿Qué características debe poseer la capacitación a directivos?}

Debe ser heurística y problemática, más que basarse en exposición de conceptos e ideas. El aprendizaje memorístico, no es lo más importante sino el cambio de conducta para reflexionar ante un problema, buscar las experiencias de avanzada en ese campo, obtener información, utilizar el pensamiento colectivo, seleccionar datos, valorar las opciones y encontrar la mejor solución. De ahí que el tratamiento especializado en la educación de directivos tiene requerimientos específicos:

$>$ No es espontáneo, requiere un plan cuya ejecución garantice el equilibrio de la organización con su entorno;

$>$ Tiene carácter diferenciado, por lo que debe basarse en un estudio de las necesidades a cada nivel: individual, grupal y organizacional;

$>$ Se orienta a la pertinencia de los recursos humanos y los cualifica de acuerdo a las necesidades del cambio organizacional;

$>$ Tiene carácter práctico, porque se basa en situaciones reales asociadas al entorno laboral, que propician hechos, habilidades y actitudes que garantizan el objetivo;

$>$ La base organizativa es el trabajo en grupos, que facilita el incremento de las ideas lógicas y creativas para la identificación, evaluación y surgimiento de alternativas;

> Retroalimenta a la organización y posibilita una continúa autovaloración que sirve de motivación para el cambio de su modelo de comportamiento:

Aprovecha el efecto experiencia. Se aprende haciendo, aprender es parte del trabajo que se perfecciona;

Requiere evaluación sistemática: de la brecha entre el estado deseado y el real de la organización debe existir un constante diagnóstico y pronóstico del comportamiento para luego realizar los ajustes a las estrategias de aprendizaje.

La evaluación de la capacitación a directivos debe producirse con un carácter permanente y sistemático; debe integrarse al sistema con el objetivo de medir la calidad de la capacitación y la influencia que ejerce en la masa de directivos, posibilitar el nexo lógico entre los distintos elementos del sistema y comparar los resultados del proceso con sus objetivos; observando qué necesidades de aprendizaje están satisfechas y cuáles no.

La capacitación se rige por principios derivados de las leyes generales del aprendizaje, en particular de la educación de adultos y de las características psicosociales que conforman a estas personas cuando desempeñan actividades de dirección.

\subsection{Enfoque sistémico en el proceso de capacitación de los Directivo}

Uno de los métodos más habitualmente aceptados y utilizados en los procesos investigativos y de dirección de los fenómenos es el enfoque sistémico, aplicable a la teoría general de sistemas.

Una definición que expresa la dimensión del término y su carácter complejo es la que ofrece Carlos Álvarez [1998:14] al definirlo como un conjunto de elementos cuyas relaciones son de un orden tal que posibilitan manifestar determinadas cualidades, propiedades totalizadoras que no se ofrecen mediante la mera suma de esos elementos. 
Analizando detenidamente esta definición resulta factible resaltar que un sistema es el resultado de la interacción y nexos que se establecen entre los elementos que lo componen, en el que cada uno realiza una función en correspondencia al lugar que ocupa en el todo. Cada elemento se analiza como una parte del todo y responde al tipo de problema a que se enfrenta y resuelve, determinando su organización y estructuración.

Esta investigación asume que en la capacitación a directivos es imprescindible observar un enfoque de sistema ya que cada una de las variables que integran el proceso tienen expresión solamente en su relación e interdependencia con las demás; así -por ejemplo- el contenido seleccionado tiene sentido si es determinado a partir de las necesidades de aprendizaje de los cursistas y las metas de las organizaciones, que a su vez están en dependencia del desarrollo interno y externo que tenga la organización de procedencia. Del mismo modo ocurre con el claustro, el que al momento de su selección no solo tiene en cuenta el contenido que se impartirá, sino también los cursistas a quienes va dirigida la capacitación. Otro punto de vista es la relación de todas las variables con el desempeño mediato y futuro, según el cual la validación final de la capacitación está dada por el impacto registrado en el área de desempeño profesional del egresado.

\subsection{El cambio organizacional}

Las condiciones dentro y fuera de las organizaciones empresariales se transforman de manera rápida y profunda. En tal sentido los siguientes aspectos también cambian: las condiciones económicas de manera dinámica, la disponibilidad y costo de los materiales, el valor del dinero, la innovación tecnológica y de los productos, las normas gubernamentales y la orientación de los mercados.

Pero también están cambiando los trabajadores porque: poseen un mayor nivel escolar, conocen mejor la importancia de los valores humanos, cuestionan la autoridad, conocen mejor sus derechos, buscan que se les respete su individualidad y tratan de mejorar la calidad de vida laboral.

Los avances tecnológicos, las normas gubernamentales, las fluctuaciones del mercado, los derechos y preferencias de los trabajadores, y los factores competitivos del entorno, indican con claridad que la variable CAMBIO es imprescindible en las organizaciones empresariales, en particular para sus directivos, pues entraña la necesidad general de una transición de un modelo burocrático de decisión y control, hacia otro donde prima el conocimiento y la creatividad, lo que algunos autores llaman gerencia de innovación.

Pero una cosa es la necesidad de cambiar y otra es la posibilidad del cambio. La primera está condicionada por factores externos e internos y sobre todo por la visión proyectiva de la organización, sus debilidades y fortalezas; el entorno, las particularidades de la actividad que realiza, su sistema de valores y su cultura, sus estrategias, y -sobre todo- por la preparación del personal y los directivos para reconocer la necesidad de cambiar. Las estrategias de cambio deben orientarse en alguna de estas direcciones.

El conocimiento y la necesidad de desarrollar habilidades para el cambio a nivel organizacional son imprescindibles.

El territorio con organizaciones que aprenden del cambio a cambiar se dota de ventajas competitivas que le marcan pautas futuras y cuya fuente es inagotable: el aprendizaje organizacional continuo.

Enfrentar y aprender el cambio dentro de las organizaciones es necesario sustentarse de un proceso de aprendizaje continuo para que estas sean competitivas. Dixon N. (1994) los consumidores satisfechos requieren el un proceso de aprendizaje a nivel individual, de sistema y de grupo, para transformar de forma continua en la organización. Diríamos... satisfacer dentro y fuera de los implicados.

Rabazza [2003] la consecuencia en el rendimento de sus organizações que ha de produzir la capacitación y el aprendizaje, define como el efecto en los individuos. 
A partir de las definiciones anteriores se asume que al hacer referencia al impacto de la capacitación se considera la existencia entre otros- de los rasgos siguientes:

$\checkmark \quad$ Expresa una relación causa-efecto entre la capacitación, el comportamiento en el desempeño profesional de sus participantes y los resultados organizacionales.

Son significativos los cambios quando tienen un carácter duradero

Los cambios cualitativos operados en las personas y las organizaciones beneficiadas por la acción de capacitación y cuantitativos, con causalidad de vinculo directo o indirecto, este juicio sobre la dinamica reflete en la evaluación del impacto [Cabrera, 2003].

Tiene el objetivo de determinar -en su forma más general- si el programa produjo los efectos deseados en los cursistas e instituciones, y si esos efectos son atribuibles a la intervención del programa. Para examinar consecuencias sean positivas o negativas no previstas en los beneficiarios, solo es posible hacerlo con la evaluaciones del impacto.

El escenario contractual, o simulado alternativo deve estimular la evaluación del impacto asegurando un rigor metodológico seguro.

Para apreciar con mayor claridad los cambios como resultado de las mediciones de la capacitación, los programas deben terminar, para determinar las diferencias entre estado detectado al diagnóstico y el existente en un período prudencial. [1990] Según Smith (42) por lo menos ser repetida dos veces. Un otro enfoque necesario para analizar la capacitación del impacto, que ha sido obviado; es la valoración de los cambios de aptitudes y pontualmente de las transformaciones actitudinales quando son adquiridos nuevos conocimientos por los directivos capacitados de la organización.

El juicio integrado, de la organización., de los cambios cuantitativos y cualitativos operados en las personas y sistémico, resultan de la evaluación del impacto.

Por la multiplicidad de criterios sobre el impacto de la capacitación, se propone un modelo que incluye elementos aceptados y que deben tenerse en cuenta. El mismo abarca tres niveles o momentos fundamentales de análisis, y son, entre otros:

1. La evaluación del proceso de capacitación: antes, durante, al final y un tiempo después.

2. La evaluación de la incidencia de la capacitación en el individuo:

- ¿ El trabajo de dirección qué le aportó ?

- ¿Cuál es el crecimiento y desarrollo individual teniendo en cuenta las necesidades de capacitación, la correspondencia entre el proceso y la satisfacción de las necesidades?

- Satisfacción: con el sistema de capacitación, las acciones y posibilidades de aplicación de los conocimientos.

- Relaciones con jefes, subordinados y homólogos; con los recursos y clientes.

3. Impacto organizacional:

- Indicadores de eficiencia: costos, rechazos y índice de desperdícios, aprovechamiento del fondo de tiempo, rotación de personal y fluctuación, Rentabilidad, volumende producción y otros;

- Indicadores de eficacia: Participación en eventos, innovaciones tecnológicas, en particular indicadores específicos de la función;

- Clima laboral;

- Satisfacción al cliente;

- Marcas, patentes. 
Como se demuestra, Las propuestas y múltiples variados análisis que se realizan tiene como objetivo de establecer un modelo de capacitación en la evaluación de las organizaciones; sin embargo, la problemática presenta dificultades ocultas, que llegan a convertirse en barreras para obtener información acertada acerca de los beneficios de la capacitación.

Una de esas barreras es valorar el impacto únicamente por los resultados económicos de la organización, estos están sujetos a múltiples influencias externas e internas, que no se resuelven sólo por la capacitación.

Para mejorar el desempeño individual y organizacional, otra condición que no debe soslayarse es la disposición del individuo a socializar el conocimiento adquirido e invertirlo.

Es imposible hablar de evaluación del impacto desde una única perspectiva porque, como se ha presentado, se trata de un proceso multifactorial y multicausal.

En tal sentido existen otros aspectos que deben ser evaluados en toda acción de capacitación como el diseño del programa durante el evento, la ejecución del mismo, sus contenidos y métodos, resultados de la acción de capacitación después de resumir las actividades relacionadas con el diseño de este dispositivo, los efectos y aplicación de lo aprendido en su puesto de trabajo y el impacto o cambios experimentados en el comportamiento individual, y en el desempeño organizacional, y su influencia en la sociedad.

Cuando se habla de impacto de la capacitación se refiere a una situación que produce un conjunto de cambios significativos y duraderos, positivos o negativos, previstos o imprevistos, en la vida de las personas, sus organizaciones y la sociedad.

Las visiones sobre impacto apuntan a que su evaluación es siempre un empeño difícil, pero necesario, en el cual debe considerarse la complejidad de la relación entre la acción de capacitación y las personas afectadas. Por ello debe distinguirse lo significativo para ponderar convenientemente la importancia de los cambios a partir de un sistema de referencia compartido.

La diversidad de enfoques permite constatar que la evaluación del impacto debe hacerse desde una perspectiva multifactorial que integre las personas, los objetivos, las acciones capacitadoras y la importancia de los cambios operados; comparado con un sistema referencial compartido y establecido de antemano.

La evaluación del impacto debe permitir: establecer la utilidad de la capacitación durante su ejecución, para la capacitación debese definir bien las orientaciones y el seguimiento, para mejorar la calidad de las acciones futuras.

Conocer con precisión el impacto de la capacitación requiere una adecuada recopilación y valoración frecuente de datos que suministren informaciones sobre la calidad de la información, la calidad de la valoración de los resultados y de la identificación de los efectos, la calidad de variables e indicadores, de acuerdo al sector o rama de la organización y la calidad de la síntesis y el análisis sistémico.

Todos deben responder a una proyección en sistema, cuyos resultados deben analizarse teniendo en cuenta multiplicidad de variables que abarquen toda la realidad organizacional.

A continuación se refieren algunos autores que han abordado el estudio relacionado con la medición del impacto de la capacitación.

Ravitsky [2002] Los quatro niveles de medición del impacto son::

- Al final de la acción encuesta de satisfacción;

- Objetivos logrados pedagógicamente;

- Puesta en práctica de las competencias en la situación profesional;

- El desarrollo de la unidad o de la empresa, depende del papel de la capacitación. 
El análisis a propósito de esta investigación demuestra lo acertado de conceptualizar la medición del impacto de la capacitación como los resultados obtenidos después de evaluar el proceso pedagógico y la aplicación de las competencias adquiridas en el puesto de trabajo.

En el análisis a lo planteado por este autor se aprecia que no tiene en cuenta la personalidad estratégica diferenciada, ni los objetivos organizacionales, que condicionan que cada unidad desarrolle independientemente sus propias variables; esto permite que aumente el conocimiento de lo realmente importante. Una misma variable puede asumirse como referencia, pero no definitiva, porque se corre el riesgo de no obtener la información necesaria que responda a las cuestiones claves de una organización. Además el autor obvia de igual manera la trascendencia que un adecuado dispositivo de formación tiene para la sociedad.

Esto es importante al definir las variables para medir el impacto de la capacitación; puesto que limitarlas a su expresión financiera soslayaría otros aspectos organizacionales. Según Eroles [2000] los sistemas de contabilidad no son buenos para medir activos no monetarios.

Las organizaciones actuales son instituciones repensadas, dotadas de capacidad distintiva como resultado del conocimiento y la información; cada día se demuestra que el valor de una empresa se relaciona más con los activos intangibles que con los tangibles, sobre los cuales se hacía la valoración, según patrones tradicionales.

En las afirmaciones anteriores aparecen elementos novedosos para la valoración del impacto de la capacitación. No se trata de desechar las informaciones contables, pero no de manera exclusiva; puesto que unido a ellos aparecen otros aspectos de la vida organizacional que en su conjunto podrán brindar datos verídicos, integrados por la adquisición de nuevos conocimientos de los directivos, que deben aplicarlos o trasladarlos a su organización. Debe medirse la contribución del conocimiento más allá del capital estructural. El aprendizaje es lo que genera nuevos modos de entender la empresa.

El seguimiento y la evaluación de los cambios que se verifican a nivel individual y de la organización en general es resultado de la evaluación objetiva de la contribución de la capacitación al logro de las metas organizacionales.

\section{Resultados para medir el impacto gerencia empresarial y administración pública}

Segundo el uso de la siguiente escala:

1. Muy bajo: no hubo ninguna satisfacción;

2. Bajo :no hubo prácticamente satisfacción;

3. Medio: si no está del todo satisfecho;

4. Alto: si está satisfecho, aunque considera que existen algunos elementos que pueden ser mejorados;

5. Muy alto: si existe una total satisfacción.

Después de medido el impacto del Diplomado que fue el empleado como base para la aplicación de los indicadores elaborados se pudo comprobar que:

\section{Variable $n^{\circ} 1$ Contenido}

Indicador \#1. 1 El 84 \% (21) de los estudiantes encuestados están satisfecho con la estructuración de los módulos, (por tanto es alto).

Indicador \# $1.2 \mathrm{El} 88 \%$ (22) de los estudiantes están satisfecho con el diseño del Diplomado, (entonces es alto). Indicador \# 1.3 El 96\% (24) de los estudiantes expresa satisfacción con la calidad de la preparación recibida. (Se considera muy alto).

Como se puede apreciar en la variable \#1(Contenido), al ser promediada obtuvo 4,4 puntos, lo que indica que alcanzó un alto impacto. Los cursistas refirieron en los instrumentos aplicados que los módulos fueron organizados coherentemente y que 
estos en su concepción estuvieron en función de sus principales necesidades de aprendizaje. Además se comenzó por darles asignaturas que estaban dirigidas a enriquecer su cultura general integral haciendo especial énfasis en el quehacer socio- histórico cultural, aspecto que le da la posibilidad a los cursistas de tener un conocimiento más integral del territorio y los dos módulos restantes estuvieron dirigidos al fortalecimiento de su preparación en los componentes económico, de dirección, técnico profesional.. L Los cuales los ponen en condiciones de conducir de forma acertada un colectivo laboral, un grupo de trabajo o tener un mejor desempeño.

\section{Variable $n^{0} 2$ Claustro de profesores}

Indicador \# 2.1 El 92 (23) \% de los estudiantes refiere estar satisfecho con la calidad del proceso docente educativo, (entonces se considera es muy alto);

Indicador \# $2.2 \mathrm{El}$ 40\% permaneció laborando en las tres ediciones, (entonces se considera medio;

Indicador \# $2.3 \mathrm{El} 30 \%$ del claustro tenía la Categoría principal es por ello que se considera bajo;

Indicador \# $2.4 \mathrm{El} 50 \%$ del claustro cuenta con Título Académico y Científico, (entonces se considera alto).

En el caso de la variable \# 2 (Claustro de profesores), alcanza 3,4 puntos, lo que es indicador de un bajo impacto.

Aunque se alcanzan altos niveles de satisfacción con la calidad del proceso docente educativo, se considera pertinente que se revise lo relacionado con la estabilidad en el claustro porque esto garantiza la consolidación y profundización en los contenidos que se imparten y un mejor perfeccionamiento de los programas a partir de las experiencias que adquiere el profesor y aportan los cursistas como agente activo del proceso. Por otra parte se hace necesario continuar trabajando en la elevación de la categoría docente y académica de los profesores que trabajan precisamente en el postgrado, porque éste exige que los profesores tengan un mayor nivel, máxime cuando se trata de los profesionales que tendrán a cargo alguna función de dirección en diferentes organizaciones y niveles jerárquicos.

\section{Variable $n^{\circ} .3$ Cursistas}

Indicador \# 3.1 El de $90 \%$ (27) de los profesores plantea que hubo una adecuada selección de los cursistas, (se considera muy alto);

Indicador \# 3.2 Se alcanza un 4,5 como promedio en cada edición entonces, (se considera muy alto);

Indicador \# $3.3 \mathrm{El} 64 \%$ de los egresados fueron promovidos a cargos de dirección, (se considera medio);

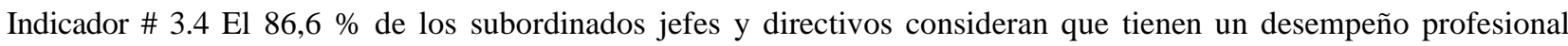
adecuado, (entonces se considera alto);

Indicador \# 3.5 El $85 \%$ de los empleadores expresa satisfacción con la calidad de la preparación mostrada por los egresados en el desempeño de sus funciones (se considera muy alto);

El promedio de la variable \# 3 (Cursistas), alcanzó 3,6 lo que indica que fue de medio impacto. La razón principal se ubica en la promoción de los cursistas una vez egresados, por la cual resulta conveniente se revise por parte de los responsabilizados con la atención de los directivos el comportamiento de este indicador ya que en ocasiones no se aprovecha consecuentemente el potencial con que se cuenta para ocupar cargos de dirección, atendiendo a que precisamente este se curso se instrumentó por la falta de preparación de los directivos en el territorio. 


\section{Variable no. 4 Métodos y medios Empleados:}

Indicador \# 4.1 El 96\% de los cursistas expresan que se aplicó la dirección participativa en el curso (se considera muy alto);

Indicador \#4.2 El $92 \%$ de los estudiantes expresan haber resuelto situaciones problemáticas dentro de las clases, (entonces se considera muy alto);

Indicador \# 4.3 El $92 \%$ de los estudiantes utilizó las TIC, (entonces se considera muy alto);

En el caso de la variable \# 4 (Métodos y medios Empleados), se promedio 4,5 puntos lo que indica que fue de muy alto impacto;

Los estudiantes plantean que la aplicación consecuente de los conocimientos y habilidades adquiridas a situaciones problemáticas le permite formarse como profesionales capaces de descubrir las problemáticas que sistemáticamente se presentan en las organizaciones y por supuesto buscarle la solución más atinada en cada caso, haciendo uso para ello de las técnicas modernas de la dirección empresarial adecuándolas a las características de cada organización y basadas en las mejores y más avanzadas prácticas contemporáneas.

Haciendo una Evaluación General (Medición Integral de impacto) del Diplomado el promedio arrojó 4,2\% lo que indica que este tiene un alto impacto que se refleja básicamente en el alto nivel de satisfacción de los cursistas con el proceso docente educativo. No obstante, a la evaluación obtenida por el curso se aprecia que existen dos variables (2 y 3 ) que deben ser revisadas para su ulterior tratamiento.

\section{Conclusiones}

La evaluación del impacto de la capacitación a directivos es una necesidad actual para hacerla más eficiente con el correspondiente ahorro de tiempo, satisfacción y transformación de los sujetos implicados, en el desempeño de sus funciones directivas.;

La aplicación de un enfoque sistémico, así como la de los métodos modernos de la capacitación a directivos constituye un objetivo esencial en la elevación de su calificación directiva y especializada;

Para que la evaluación sea apropiada y certera deben existir objetivos medibles, con indicadores significativos y criterios determinados;

La evaluación del impacto de la capacitación a los directivos es un tema en el que se debe continuar profundizando en función un mejor apoyo al proceso de perfeccionamiento de la referida capacitación.

Esta investigación constituye un material de consulta para profesores, metodólogos y directivos porque en ella se ofrecen instrumentos para evaluar el impacto de la capacitación de los directivos a partir de un sistema de indicadores.

\section{Agradecimentos}

Nuestro agradecimiento a todos que participaron y contribuyeron directa o indirectamente, que nos permitió llevar adelante este Artículo.

\section{Referencias}

Álvarez, Z.C. M. \& González, E. M. (1998). Lecciones de Didáctica General. Didácticas Magisterio.

Álvarez, Z. C. M. \& Sierra, V. (1998). Metodología de la Investigación Científica. Revista La Habana: Universidad de la Habana.

Álvarez, Z. C. M. (1996). Hacia una Escuela de excelencia: La concepción didáctica de la Educación Superior cubana. La Habana, Academia. http://revistas.udea.edu.co 
Research, Society and Development, v. 11, n. 2, e34511221245, 2022

(CC BY 4.0) | ISSN 2525-3409 | DOI: http://dx.doi.org/10.33448/rsd-v11i2.21245

Arias, M. (1988). Los cuadros, representantes de vanguardia del estado. Revista El Economista No 7, p. 56-72.

Buckley, R. y Caple, J. (1991). La formación. Teoría y Práctica. Madrid: Díaz de Santos S.A., p. 7-38, 185, 207-209

Baker, J. (2000). Evaluación del impacto de los proyectos de desarrollo en la pobreza: manual para profesionales. Banco Mundial, Washington D.C. http://www.cddhcu.gob.mx/bibliot/publica/inveyana/pdf.

Cabrera, J. (2000). Sociedad del conocimiento: ¿somos competentes? Soporte magnético. UNAH

Calderón, H. (1995). Manual para la administración del proceso de capacitación de personal. Editorial Limusa, S.A. de C.V. Grupo Noriega Editores, México, D.F.-, p.18

Carballal, E., Díaz, C. \& Rodríguez, J. (1999). La calidad aplicada al diseño de cursos de capacitación a dirigentes. Folletos Gerenciales (Cuba). No. 7, Julio, CCED-MES

Carballal, E., Díaz, C. \& Rodríguez, J. (1999). La calidad aplicada al diseño de cursos de capacitación a dirigentes. Folletos Gerenciales. (Cuba) Año V, No 7, Septiembre, CCED-MES p. 10-19

Carballal, E., Díaz, C. \& Rodríguez, J. (1999). La calidad aplicada al diseño de cursos de capacitación a dirigentes. CCED-MES, Folletos Gerenciales (Cuba). No 7: CCED - MES, 99, p. 10-19

Casanova, A. \& Diez, A. (1996). El desarrollo del sistema empresarial en el contexto de las transformaciones económicas. Ponencia del Seminario sobre Derecho Económico y Cambios en el Marco Jurídico. - La Habana, p. 1-9

Codina, A. (1998). “¿Qué hacen los directivos y qué habilidades necesitan para un trabajo en efectivo?” Folletos Gerenciales (Cuba). Año II, No. 4, Abril, CCED-MES.

Columbié, M. (1999). "La superación de los cuadros en Cuba: sus retos ante el Tercer Milenio”. Folletos Gerenciales (Cuba). No.1, Enero, CCED-MES.

Drucker, P. (1973). La gerencia. Tareas, responsabilidades y práctica. El ateneo, Buenos Aires.

Estudio internacional sobre criterios e indicadores de calidad de las universidades. Agencia de Calidad, Acreditación y Prospectiva de las Universidades de Madrid (ACAP) (2006). http://www.madrid.org/bvirtual/BVCM001820.pdf.

García, F. (2001). La capacitación en dirección. Herramienta para el cambio estratégico en la organización: un sistema para la formación y superación de los cuadros y sus reservas. Folletos Gerenciales (Cuba). Año V, No. 9, Septiembre, CCED-MES

García, G.( 2001). La formación: una moneda con sus dos caras. Folletos Gerenciales (Cuba). Año V, No. 7, Julio, CCED-MES.

Goleman, D., Boyatzis, R. y Mckee, A. (2002). El líder resonante, crear más. El poder de la inteligencia emocional. España, Ed. Plazas \& Janés, S.A.

Kubr, M. y Col. (1984). La administración de una institución de desarrollo administrativo. Ginebra, Ed. Oficina internacional del Trabajo.

Gandarilla, M. S. \& Col. (2001). Preparación y desarrollo de una reserva estratégica de cuadros para el territorio: una alternativa viable que se aplica en Granma. Folletos Gerenciales (Cuba). Año V, No.5, Mayo, CCED-MES.

Mariño, R. Y Col. (1999). Consideraciones para la elaboración de una estrategia individual de desarrollo de cuadros y reservas. Experiencias e impacto de su aplicación. Folletos Gerenciales (Cuba). Año V, No. 6, Junio, CCED-MES.

Mendoza, A. (1986). Manual para determinar necesidades de capacitación. México DF, Trillas.

Pinto, M E. (2001). Algunos apuntes sobre la medición de impacto de la capacitación a dirigentes. Folletos Gerenciales (Cuba). Año V, No.1, Enero, CCEDMES.

Rabaza F. Y Col. (2000). Valoración de la capacitación. Vías para su ejecución. Folletos Gerenciales (Cuba), año IV, No. 4, Abril, CCED-MES.

Rabaza, F., Trenal, T. y Reyes, E. (2003). La medición del impacto de la capacitación: en caso de un Diplomado. Folletos Gerenciales (Cuba), Año V, No 9, septiembre, CCED-MES.

Valiente, P. (2001). El proceso de enseñanza aprendizaje de los dirigentes: características y exigencias. Folletos Gerenciales (Cuba), Año V, No. 4, Abril, CCED-MES 